\title{
A Review on Wastewater Treatments of Lead-Zinc Concentrator in China
}

\author{
$\mathrm{Xu}$ DONG \\ Faculty of Land Resource Engineering, \\ Kunming University of Science and Technology \\ Kunming, Yunnan province, China \\ a451184428@qq.com \\ Hai-lin LIU \\ Faculty of Land Resource Engineering, \\ Kunming University of Science and Technology \\ Kunming, Yunnan province, China
}

\section{Yi PEI}

Faculty of Land Resource Engineering, Kunming University of Science and Technology Kunming, Yunnan province, China

\author{
Si-qing LIU* \\ Faculty of Land Resource Engineering, \\ Kunming University of Science and Technology \\ Kunming, Yunnan province, China \\ e-mail: 451184428@qq.com

\section{Dan WANG} \\ Faculty of Land Resource Engineering, \\ Kunming University of Science and Technology \\ Kunming, Yunnan province, China
}

\begin{abstract}
During the last decade, lead-zinc mining industry in China has made a significant progress and China has become one of the most important producers in the world. In this paper, the recent developments in both treatments and reuse of wastewater of lead-zinc concentrators in China are briefly reviewed. Current situation of the main treatment methods like self-purification, coagulation sedimentation, adsorption, biofilm process, constructed wetland are introduced. And different applications of wastewater recycling are shortly discussed.
\end{abstract}

Keywords-Lead-zinc concentrator; Wastewater; Treatment; Recycling.

\section{INTRODUCTION}

Lead-zinc mineral resources as the predominant mineral resources are widely found in China. Production, consumption and exports of lead and zincrank the first in the world. Lead-zinc ore dressing is an important segment in the process of lead-zinc production, providing raw materials for the industry, however, producing a large amount of wastewater in processing the lead-zinc ores. With the implementation of Chinese policies on water-saving for emission reduction and wastewater reutilization, concerns about the wastewater treatment of lead-zinc concentrators have been heightened in recent years, so as to comply with the construction of "two oriented society" (resource-conserving \& environmental-friendly society), to meet the requirements of the increasing environmental protection.

The direct discharge of wastewater in the tailings not only consumes a large amount of water resources, but also contains an amount of flotation reagents, which are characterized by complex composition, harmful components and strong odor. It can cause serious impacts on the environment. In particular, lead in the tailings can be accumulated in the environment or animals' body, and it would lead to a long-term impact on human body's nervous system.

\section{WASTEWATER SOURCE}

The complex composition of wastewater and the interaction between the materials make it difficult to treat the wastewater. At present, the main methods of wastewater treatments of lead-zinc concentrators mainly deal with coagulation sedimentation, adsorption, biofilm process, constructed wetland, etc.

\section{WASTEWATER TREATMENTS}

The complex composition of wastewater and the interaction between the materials make it difficult to treat the wastewater. At present, the main methods of wastewater treatments of lead-zinc concentrators mainly deal with coagulation sedimentation, adsorption, biofilm process, constructed wetland, etc.

\section{A. Coagulation and Sedimentation}

In the presence of coagulant, suspended solids, heavy metal ion and other harmful matter get sedimented. Common coagulants are ferrous sulfate, polyalumi rlillm sulfate, polymeric ferric sulfate and alum, etc. Meanwhile, some coagulant aids are added to enhance the coagulant performance. Common coagulant aids are $\mathrm{pH}$ modifiers, aggregates structures modifiers and polyacrylamide (PAM), etc. Coagulation method is often combined use with adsorption and neutralization method. In the process of coagulation sedimentation-adsorption, the COD value of the wastewater can be reduced by adding an adsorbent.

The dosage of total aluminum $40 \mathrm{mg} / \mathrm{L}$, the removal rate of $\mathrm{Pb}^{2+}$ is up to $87.14 \%$. After treating the coagulating sedimentation, the activated carbon is adopted to adsorb the 
organic residues of chemical reagents in wastewater. When activated carbon is added at the dosage of $100 \mathrm{mg} / \mathrm{L}$, the removal rate of $\mathrm{Pb}^{2+}$ and organic content is up to $93.24 \%$ and $56.32 \%$, respectively. The results show that effect of flotation using the treated water, which is treated by coagulating sedimentation-activated carbon adsorption, is almost the same as that of fresh water [2].

\section{B. Biofilm Process}

Due to the fact that biodegradation method is efficient in degrading organic pollutants, without secondary pollution and low cost, much attention has been paid on this technology. L[3], studied an application of biofilm process for wastewater treatment of lead-zinc. With hydraulic retention time of 2 days, the most appealing effects of biofilm treatment of $50 \mathrm{~L}$ wastewater can be observed at $6 \sim 13^{\circ} \mathrm{C}$, while the viscose material as a carrier. The COD was maintained at $40 \mathrm{mg} / \mathrm{L}$. After treatment of biofilm, the effluent could meet the emission standard of pollutants for lead-zinc industry.

Biofilm method covers a small area, does not produce a lot of waste sludge form, avoiding secondary pollution problem. However, biofilm on the work requirements of environmental conditions are relatively harsh, and it is of poor adaptation, resulting in less current practical application.

\section{Constructed Wetland}

The constructed wetland is the focus of research in recent years. It has much advantage, such as stable water quality, low capital expenditure, easy to operate, convenient maintenance management, strong impulsion load resistance and so on. The basic principle is to achieve the wastewater resource harmlessness, using coordination roles of physical, chemical and biological ways in matrix, microorganism, animals and plants by filtration, adsorption, co-precipitation, ion exchange, plant absorption and microbial decomposition to realize efficient purification of sewage, and through the biogeochemical cycle to provide nutrients and moisture to promote plant growth.

G[4],studied the content and distribution characteristics of heavy metal from water and sediment of Fankou lead-zinc flotation concentrator in the constructed wetland. The results showed that in the wetland the heavy metal existed mainly in suspended solid fraction, as the increase of horizontal flow movement, total content of lead and zinc in water firstly increased and then decreased. The content of the two elements in sediment showed very significant negative correlation with the distance of horizontal flow movement $(\mathrm{P}<0.01)$. Heavy metal exited mainly in residual fraction in sediment and organic combination fraction.

The constructed wetland system is a complete ecosystem, it can treat the sewage, mean while, it can create a good environment, because most of the mine is located in remote areas with a good environment, therefore the wetland treatment of wastewater has very significant economic, social and environmental benefits, and it is expected to widen its application in the future.

\section{Other Treatments}

In some cases, flocculating sedimentation tests of JCSS flocculant were conducted with wastewater from lead-zinc concentrator is almost the same as using clean water. Industrial test proves that the wastewater treated by JCSS flocculant can reach the requirements of reused water recycled from the wastewater [5]. The strong acid cation exchange fiber was used to treat the wastewater from lead-zinc mine containing $\mathrm{Fe}, \mathrm{Zn}, \mathrm{Cd}, \mathrm{Pb}$ and other heavy metal. The results show that iron removal rate is higher than 99.9\% after pretreatment by $\mathrm{NaOH} / \mathrm{Ca}(\mathrm{OH})_{2}$ (1ime cream) . Compared with $\mathrm{NaOH}$ pretreatment methods, because of a large number of $\mathrm{Ca}, \mathrm{Mg}$ ions existence in the wastewater, the unit treatment capacity by $\mathrm{Ca}(\mathrm{OH})_{2}$ pretreatment is reduced by $33 \%$, and followed by ion exchange fiber column treatment, $\mathrm{Cd}, \mathrm{Zn}$ content in effluent can reach Class III of Surface Water prescribed by Chinese National Standard [6].

\section{WASTEWATER REUSE}

Basically, after purification treatment, the lead-zinc wastewater can meet the national emission standards. But from an economic point of view, wastewater's being directly discharged into the natural environment will inevitably lead to economic losses, and enlarge the cost of mineral processing. Therefore, at present, the wastewater of lead-zinc concentrators, even in the case of not meeting the emission standards, can also return to some specific flotation circuits directly or after a certain treatment under the condition that no influence on the mineral processing performance. So it can reduce the difficulty and cost of wastewater treatment, and can save the fresh water resources, realize the recycling of water resources and improve economic performance.

\section{A. Tailings Dam Overflow Backwater Process}

C[7], studied the total backwater process of tailings dam overflow on a lead-zinc flotation plant in Hunan. And the results showed that the effluent could be fully reused for the concentrator, the flotation operations were stable, which were similar to those of the fresh water, so the zero-discharge of wastewater could be realized.

Tailings dam overflow backwater process can not only reduce the cost of wastewater treatment with the help of self purification capacity of tailings dam, but also realize the unified scheduling of the concentrator wastewater, and this process is relatively simple, and easy to manage, thus is favored by most plants. But this process converges different sources of wastewater, resulting in a more complex composition of the reuse of the wastewater, thus may make the dressing operation unstable. Moreover, if there's long way between the tailings dam and the concentrator, the cost of pipeline transportation may be greatly increased, leading to a poor economic performance.

\section{B. Wastewater Returns to Different Circuits}

Wastewater's returning to different flotation circuits is another important process for realizing the recycling of lead-zinc dressing wastewater. The water quality of different operating points of mineral processing differs from each other. And the wastewater treated or not can return to a 
certain processing operation. Wastewater's returening system can not only promote the recycling of wastewater, but also can avoid the adverse effects of the wastewater on other mineral processing operations, and then a stable mineral processing performance would be obtained. $\mathrm{H}[8]$, studied the wastewater from various sections of flotation process, the way of staged recycling was adopted to fully recover part of the wastewater in order to reduce the wastewater to be treated and get a lower cost on the treatment without influencing the concentrate recovery rate, and remarkable economic profits and environmental benefits can be obtained, saving $¥ 270,000$ per month on the cost of wastewater.

Optimum process of Part Wastewater directly reused and Ohters treated to reuse was adopted to solve the problem that wastewater is rich in reagents, valuable and harmful substances coexist. This process can also be subdivided into 2 types: A. part of wastewater is been reused directly, the residual been treated and then returened the operation, this is definitely the development of Part Wastewater directly reused and Ohters treated to reuse; B. part of wastewater is been reused directly, the residual converges into the tailings dam to be treated at the same time.

The second type is the combination of the said two main processes of wastewater reuse, which has properties of the both. Z[9], adopted the process of part directly reuse and others after treated to reuse, and the wastewater reuse rate reached more than $85 \%$, the amount of new water was reduced and the cost of production operation was reduced as well. The staged recycling of wastewater can reduce the total water consumption. However, compared with the tailings dam overflow backwater process, this process requires more closed circuits circulation of wastewater, which make it difficult to maintain and manage the whole process.

\section{CONCLUSIONS}

Whether it is the purification technology of lead-zinc wastewater, or the recycling technology of the wastewater, both have obvious advantages and disadvantages. Make the most of these treatments would benefit both economic performance and environmental protection. So mineral processors and environmental engineers should pay attention, when in charge of process of the construction of a new plant, to the rules that are based on the long-term and overall planning, rational layout, formulate detailed feasible implementation plan; and for the transformation of the old plant process in existing technology and facilities, from the practical point of view, and map out a feasible scheme by adopting scientific processing technologies to minimize the amount of wastewater in production. Only in this way, can an economic and environmental-friendly performance of lead-zinc wastewater treatments come true.

\section{REFERENCES}

[1] L. Siqing, Modern Mining, In Chinese (2009, pp. 101-104).

[2] D. Dong, Nonferrous Metals, In Chinese (No. 3, 2012, pp. 28-31).

[3] L. Weixiong, Chinese Journal of Environmental Engineering, In Chinese (No. 1, 2014, pp. 67-71).

[4] G. Jinlin, Guangzhou Chemical Industry, In Chinese (2014, pp. 112-115).

[5] L. Shuzhong, Nonferrous Metals, In Chinese ( No. 5, 2014, pp. 85-87).

[6] Q.Zhaoke, Technology of Water Treatment, In Chinese (No. 12, 2013, pp. 99-107)

[7] C. Wei, Environmental Engineering, In Chinese (No. 3, 2011, pp. 37-39.).

[8] H. Shihe, Nonferrous Metals, In Chinese (No. 5, 2011, pp. 24-26.).

[9] Y.anfen, Yunnan Metallurgy, In Chinese (No. 3, 2013, pp. 68-72.). 